\title{
Anticonvulsant Activity of Acute and Chronic Treatment with a-Asarone from Acorus gramineus in Seizure Models
}

\author{
Qi-Xiong Chen, ${ }^{*, a}$ Jing-Kun Miao, ${ }^{b, \#}$ Chun Li, ${ }^{a}$ Xiao-Wen Li, ${ }^{a}$ Xiao-Mei Wu, ${ }^{b}$ and \\ Xiao-ping Zhang ${ }^{c}$ \\ ${ }^{a}$ Department of Neonatology, The Children's Hospital, Chongqing Medical University; Chongqing 400014, China: \\ ${ }^{b}$ Chongqing International Science and Technology Cooperation Center for Child Development and Disorders; \\ Chongqing 400014, China: and ${ }^{c}$ Key Laboratory of Pediatrics in Chongqing; Chongqing 400014, China. \\ Received April 25, 2012; accepted September 10, 2012; advance publication released online October 18, 2012
}

For centuries, extracts of Acorus gramineus have been used extensively in traditional Chinese medicine for the treatment, management, and/or control of human ailments, including central nervous system disorders such as convulsions and epilepsy. In the present study, we investigated the anticonvulsant activity of chronic treatment with the plant's major essential oil component (a-asarone, $50-200 \mathrm{mg} / \mathrm{kg}$, per os (p.o.)) against maximal electroshock seizure (MES), pentylenetetrazole (PTZ)-induced seizures in mice, lithiumpilocarpine (LI-PILO)-induced status epilepticus (SE), and spontaneous recurrent seizures (SRSs) in rats and determined whether a single acute administration of a-asarone at various doses could produce anticonvulsant activity. As the standard antiepileptic drugs used, chronically administered a-asarone (50-200 mg/ $\mathrm{kg}$, p.o. $)$ significantly delayed $(\boldsymbol{p}<\mathbf{0 . 0 5})$ the onset of, and antagonized maximal electroshock seizure and PTZ-induced seizures. Chronically administered a-asarone (50-200 $\mathrm{mg} / \mathrm{kg}$ ) also profoundly antagonized LI-PILO-induced seizures. The SE incidence, SE latency and seizure severity as well as mortality were significantly reduced after treatment with a-asarone at different doses. Higher doses of a-asarone (100-200 $\mathrm{mg} /$ kg) significantly reduced spontaneous recurrent seizure incidence, severity, and seizure frequency during treatment in LI-PILO-induced SRSs rats. On the other hand, a single acute administration of a-asarone (50-200 $\mathrm{mg} / \mathrm{kg}$ ) produced weak anticonvulsant activity in MES and PTZ-induced seizures. The results of this laboratory animal study indicate that chronically administered a-asarone possesses anticonvulsant activity in the mammalian experimental model used, and thus suggest that a-asarone may be used as a natural supplementary remedy in the management of convulsions and epilepsy.

Key words anticonvulsant activity; a-asarone; Acorus gramineus; seizure; animal model

Epilepsy is a chronic neurological disorder characterized by unprovoked seizures that affects $0.5-1.0 \%$ of the population. ${ }^{1)}$ However, with the available antiepileptic drugs on the market, such as phenobarbital, phenytoin, carbamazepine, sodium valproate, benzodiazepines, and ethosuximide, although about $70-80 \%$ of the people with epilepsy achieve satisfactory seizure control, nearly $20-30 \%$ of patients who develop partial and secondary generalized seizures are still refractory to treatment with the current antiepileptic drugs (AEDs). ${ }^{2}$ Furthermore, the dose-related neurotoxicity and other side effects associated with established antiepileptic drugs limit their clinical use. As a consequence, it is imperative to develop new AEDs with high efficacy and less adverse effects. ${ }^{3)}$

Recently, the alternative management of epilepsy with traditional Chinese medicines has become attractive to patients as well as physicians in both China and Western countries. ${ }^{4)}$ In traditional Chinese medicine, the roots and rhizomes of Acorus gramineus have been recommended for centuries for the treatment of human ailments, including central nervous system disorders such as convulsions and epilepsy, or in combination with other medical herbs for improvement of learning and memory. ${ }^{5,6)}$ This herbal drug has been reported to be responsible for various pharmacological actions on the central nervous system. Aqueous and methanol extracts of Acorus gramineus have been shown to antagonize pentylenetetrazole (PTZ)-induced convulsion, potentiate pentobarbital-induced

The authors declare no conflict of interest.

${ }^{\#}$ Equal contribution with first author. sleeping time, induce sedation, and decrease spontaneous activity in mice. ${ }^{7,8)}$ In addition, the extracts of composite drugs containing Acorus gramineus as a main component are used for improvement of learning and memory and for stroke. ${ }^{9,10)}$ However, the essential oil of Acorus gramineus has not been thoroughly evaluated. The rhizomes and leaves of Acorus gramineus are known to contain $0.11-0.42 \%$ of essential oil consisting of 30 kinds of compounds which may affect the central nervous system (CNS) after direct inhalation of its fragrance. ${ }^{11,12)}$ To date, only a few papers have reported on the sedative or activating properties of some essential oils and their constituents in animals under standardized experimental procedures. ${ }^{13-16)}$ However, the major components possessing the anticonvulsant effects are still poorly understood.

a-Asarone (trans-1-propenyl-2,4,5-trimethoxy-benzene) is the active principle of Acorus gramineus (for its structure, see Fig. 1). Many of the studies on the anticonvulsant activity of a-asarone yielded marginal effects. However, in these studies, a-asarone was acutely administered. ${ }^{17)} \mathrm{A}$ recent study that examined twice daily essential oil inhalation for over 4 weeks found that chronically inhaled essential oil provided increased anticonvulsant activity in mice. ${ }^{16)}$ The present study investigated whether the anticonvulsant activity of a-asarone was dependent on chronic administration and determined whether single acute doses of a-asarone had anticonvulsant activity in the different experimental seizure models, including the maximal electroshock seizure (MES) test, subcutaneous pentylenetetrazol seizure (scPTZ) test, and lithium-pilocarpine (LI-PILO) model. 


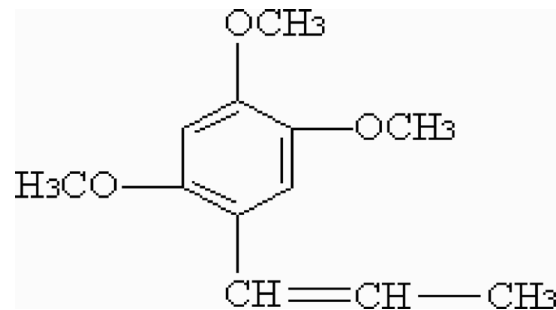

Fig. 1. Chemical Structure of a-Asarone

\section{MATERIALS AND METHODS}

Animals and Experimental Conditions Male Swiss mice weighing 20-30 g, and male Wistar rats weighting 200-250 g, were used. The animals were kept under controlled environmental conditions $\left(23 \pm 2{ }^{\circ} \mathrm{C} ; 55 \pm 20 \%\right.$ humidity; $12 \mathrm{~h}$ light/dark cycle, lights on at $6: 00 \mathrm{~h}$ ) with free access to standard laboratory diet and tap water. Animals were allowed to acclimate to the laboratory environment for $\geq 5 \mathrm{~d}$. All animal experiments were performed following approval of the Committee of Experimental Animal Administration of the University and were in accordance with the National Institutes of Health Guide for Care and Use of Laboratory Animals.

Materials a-Asarone (trans-1-propenyl-2,4,5-trimethoxybenzene) was purchased from Shengyang Aisheng Pharmaceutical Co., Ltd. (Shengyang, China). Diazepam was purchased from Hubei Pharmaceutical Factory (Hubei, China). Methylscopolamine-bromide, pentylenetrazol, lithium chloride, and pilocarpine nitrate were purchased from Sigma (U.S.A.). Valproic acid (VPA) (Hunan Xiangzhong Pharmaceutical Co., Ltd., China), Carbamazepine (CBZ) (Shanghai Sunve Pharmaceutical Co., Ltd., China) and Clonazepam (CNP) (Xuzhou Enhua Pharmaceutical Co., Ltd., China) were used as reference anticonvulsant drugs for comparison. VPA was dissolved in saline, while CBZ and CNP were suspended in saline. Three control AEDs were prepared each day as fresh solutions or suspensions and administered orally in a volume of $10 \mathrm{~mL} /$ $\mathrm{kg}$ body weight.

Acute Toxicity Test The acute toxicity and lethality of a-asarone were determined in mice according to a modified method of Lorke. ${ }^{18)}$ Mice were randomly divided into 8 groups of 8 animals each for a total of 64 mice. Stepwise, graded doses of a-asarone (10, 20, 40, 100, 200, 400, $1000 \mathrm{mg}$ / $\mathrm{kg}$ ) were orally administered to the mice in each of the test groups. All mice in the control group were treated with normal saline only. The mice in both the test and control groups were then allowed free access to food and water, and observed over a period of $48 \mathrm{~h}$ for signs of acute toxicity. The number of deaths (caused by the a-asarone in each group) within this period of time was recorded. Log-dose response plots were constructed for a-asarone, from which the median lethal dose $\left(\mathrm{LD}_{50}\right)$ of a-asarone was calculated as the geometric mean of the highest nonlethal dose and the lowest lethal dose.

Evaluation of Adverse Effects of Chronic Treatment Mice were randomly divided into 4 groups of 8 animals each for a total of 32 mice. Groups of 8 mice were used per dosage $(50,100,200 \mathrm{mg} / \mathrm{kg} / \mathrm{d})$ of a-asarone, administered twice daily for $28 \mathrm{~d}$. The control group was treated with orally administered normal saline. Animals were observed at various times after oral administration for adverse effects. Appearance and overt behavior were recorded to determine any changes in the skin and fur, eyes and mucous membranes as well as any disturbances in the respiration, circulation, autonomic or central nervous systems and behavior patterns.

Evaluation of Anticonvulsant Property. Effects of Acute and Chronic Treatment with a-Asarone on MES Test in Mice Electroconvulsions were produced by an alternating current $(0.20 \mathrm{~s}$ stimulus duration, $50 \mathrm{~Hz}$, fixed current intensity of $25 \mathrm{~mA}$, maximum stimulation voltage of $500 \mathrm{~V})$ delivered via ear-clip electrodes by a rodent shocker generator (YSD4G) (Shanghai Xinman Scientific and Educational Apparatus Co., Ltd., China). Tonic hind limb extension (hind limbs of animals outstretched $180^{\circ}$ to the plain of the body axis) was taken as the endpoint. ${ }^{19)}$

In the acute administration test, mice were divided into 4 groups of 20 animals each for a total of 80 mice. In 3 groups, a single dose $(50,100,200 \mathrm{mg} / \mathrm{kg})$ of a-asarone was orally administered $60 \mathrm{~min}$ before the electroshock to quantify the anticonvulsant effect. The control group was treated with orally administered normal saline $(10 \mathrm{~mL} / \mathrm{kg})$.

In the chronic administration test, mice were randomly divided into 5 groups of 20 animals each for a total of 100 mice. Five groups were treated with orally administered normal saline (as control), VPA $(200 \mathrm{mg} / \mathrm{kg} / \mathrm{d}$ as positive control) and a-asarone $(50,100,200 \mathrm{mg} / \mathrm{kg} /$ day $)$, twice daily, respectively, for $28 \mathrm{~d}$. Seizures were induced at $60 \mathrm{~min}$ after the last administration, and the number of animals exhibiting tonic hind limb extension and mortality were recorded. Animals failing to show tonic hind limb extension were regarded as being protected.

Effects of Acute and Chronic Treatment with a-Asarone on scPTZ Test in Mice Pentylenetetrazol (PTZ) was administered subcutaneously at $85 \mathrm{mg} / \mathrm{kg} .{ }^{20)}$ Animals were returned to their cages and observed for $60 \mathrm{~min}$ for the presence of seizures. The presence of clonic convulsion indicated successful establishment of the scPTZ model. ${ }^{21)}$ The onset of a general clonus was used as the endpoint. General clonus was characterized by forelimb clonus followed by full clonus. The time taken before the onset of clonic convulsions, the duration of clonic convulsions, and the incidence of seizure and mortality were recorded. Animals failing to show clonic seizure were scored as being protected. Animal grouping and treatment of the animals were the same as for the MES test.

In the acute administration test, the mice were given a single dose of a-asarone at the doses of 50,100 , or $200 \mathrm{mg} /$ $\mathrm{kg}, 60 \mathrm{~min}$ before the administration of PTZ $(85 \mathrm{mg} / \mathrm{kg})$. The control group was orally administered normal saline $(10 \mathrm{~mL} /$ $\mathrm{kg}) 60 \mathrm{~min}$ before the administration of PTZ $(85 \mathrm{mg} / \mathrm{kg})$.

In the chronic administration test, 5 groups were treated with orally administered normal saline (as control), CNP ( $2 \mathrm{mg} / \mathrm{kg} / \mathrm{d}$ as positive control) and a-asarone $(50,100,200 \mathrm{mg} /$ $\mathrm{kg} / \mathrm{d}$ ), twice daily, respectively, for $28 \mathrm{~d}$. Seizures were induced at $60 \mathrm{~min}$ after the last administration.

Effects of Acute Treatment with a-Asarone on LI-PILO Induced Status Epilepticus (SE) in Rats Lithium plus pilocarpine combinations were used to induce SE, because pretreatment of rats with lithium chloride allows the dose of pilocarpine to be decreased, resulting in a lower mortality and higher percentage of animals exhibiting SE. ${ }^{22)}$ Lithium chloride was injected at $3 \mathrm{mEq} / \mathrm{kg}(125 \mathrm{mg} / \mathrm{kg})$ intraperitoneally (i.p.), $18-24 \mathrm{~h}$ before the administration of pilocarpine. 
Furthermore, rats were first injected with methylscopolaminebromide $(1 \mathrm{mg} / \mathrm{kg}$, i.p.), to prevent the peripheral cholinomimetic effects of pilocarpine. ${ }^{23)}$ Pilocarpine $(40 \mathrm{mg} / \mathrm{kg}$, i.p.) was administered at $30 \mathrm{~min}$ after methylscopolamine treatment. Animals were divided into 5 groups of 20 rats each for a total of 100 rats. Five groups were treated with orally administered normal saline (as control), CBZ $(200 \mathrm{mg} / \mathrm{kg} / \mathrm{d}$ as positive control) and a-asarone $(50,100,200 \mathrm{mg} / \mathrm{kg} / \mathrm{d})$, respectively, $60 \mathrm{~min}$ before receiving pilocarpine $(40 \mathrm{mg} / \mathrm{kg})$. Approximately $30 \mathrm{~min}$ after pilocarpine treatment, the rats began to manifest motor seizures. Seizure severity was scored according to a modified Racine scale, and only motor seizures were scored. ${ }^{24)}$ Seizures were graded as follows: class III, rats displayed forelimb clonus with a lordotic posture; class IV, rats reared with a concomitant forelimb clonus; class $\mathrm{V}$, rats had the characteristics of class IV and fell down. ${ }^{25)}$ After pilocarpine injection, the animals were placed back into their plastic cages and observed in isolation for at least $3 \mathrm{~h}$. All motor seizures as well as behaviors were recorded. The incidence and onset of the motor SE, severity of seizures, and mortality were recorded. One hour after continuous motor SE, diazepam was administered $(10 \mathrm{mg} / \mathrm{kg}$, i.p.) to reduce the otherwise high mortality rate in this period. Two to three days after SE onset, the rats were fed a mixture of sweetened milk $\geq 3$ times/d.

Effects of Chronic Treatment with a-Asarone on LIPILO Induced Spontaneous Recurrent Seizures (SRSs) in Rats Twenty-four hours after SE, the rats were divided into 5 groups of 20 rats each for a total of 100 rats. Animal grouping and treatment were the same as for the MES test. CBZ $(200 \mathrm{mg} / \mathrm{kg} / \mathrm{d})$ was used as a positive control. Animal behavior was video-monitored $(12 \mathrm{~h} / \mathrm{d})$ during treatment and for $28 \mathrm{~d}$. The parameters analyzed were (a) the onset of the first spontaneous seizure, (b) incidence of seizures, (c) frequency, and (d) severity of seizures. ${ }^{26)}$ SRSs in these animals were scored according to Racine. ${ }^{24)}$ Animals failing to show class III-V seizures were regarded as being protected.

Statistical Analysis The results were expressed as the mean \pm S.E.M. values. All statistical analyses were performed using SPSS11.0 software for Windows. Differences in the seizure rate and mortality rate were assessed by the $\chi^{2}$ test. Statistically significant differences between the vehicle and a-asarone treatment groups were assessed by analysis of variance (ANOVA) followed by Dunnett's multiple comparison test. The rank sum test was used to analyze non-parametric data. Probability values of less than $0.05(p<0.05)$ were considered to be statistically significant.

\section{RESULTS}

Lethality $\left(\mathbf{L D}_{\mathbf{5 0}}\right)$ of a-Asarone The acute toxicity test showed that oral administration of a-asarone was non-toxic up to a dose of $1000 \mathrm{mg} / \mathrm{kg}$ and did not cause any death in the test groups. The oral $\mathrm{LD}_{50}$ of a-asarone in mice was therefore greater than $1000 \mathrm{mg} / \mathrm{kg}$.

Adverse Effects of Chronic Treatment with a-Asarone For mice treated with graded doses of a-asarone $(50,100 \mathrm{mg} /$ $\mathrm{kg}$ ), no overt behavioral changes were observed. For example, it did not induce any significant changes in the walking, rearing and grooming behaviors of mice. In addition, no sedation or loss of righting reflex was evident. At a higher dose $(200 \mathrm{mg} / \mathrm{kg})$, a-asarone then exerted a slightly sedative and tranquillizing action $20.3 \pm 3.8 \mathrm{~d}$ after treatment. Locomotor analysis performed after the oral administration of a-asarone $(200 \mathrm{mg} / \mathrm{kg})$ showed a slight decrease in spontaneous movement $(25.6 \pm 14.2 \%)$ as compared to the vehicle treated group $(10.3 \pm 6.8 \%)$. Our results suggest that a-asarone is relatively safe in mice.

Effects of Chronic Treatment with a-Asarone in MES Test All animals receiving vehicle showed tonic hind limb extension after electrical stimulation (Table 1). Chronic treatment with a-asarone at doses of 50,100 , and $200 \mathrm{mg} / \mathrm{kg}$ significantly decreased the occurrence of tonic hind limb extension induced by electroshock in $40 \%(8 / 20), 20 \%(4 / 20)$, and $0(0 / 20)$ of tested animals, respectively, compared to $100 \%$ $(20 / 20)$ in the vehicle treated group (Table 1). All animals were free of seizures in the VPA group $(p<0.01)$. Higher doses of a-asarone $(100-200 \mathrm{mg} / \mathrm{kg})$ significantly $(p<0.01)$ reduced the hind limb extensor phase of convulsion as compared to the vehicle treated animals. a-Asarone at doses of 50,100 , and $200 \mathrm{mg} / \mathrm{kg}$ significantly decreased the mortality induced by MES in 30\% (6/20), 10\% (2/20), and $0(0 / 20)$ of tested animals, respectively, compared to $40 \%(8 / 20)$ in the vehicle treated group (Table 1). a-Asarone and VPA $(200 \mathrm{mg} /$ $\mathrm{kg}$ ) significantly decreased the incidence of tonic hind limb extension and mortality induced by electroshock.

Effects of Acute Treatment with a-Asarone in MES Test All the mice treated with vehicle suffered seizures when submitted to the MES test (Table 2). There was no difference between abolishment of hind limb tonic extension and death in the mice treated orally with 50,100 , or $200 \mathrm{mg} /$ $\mathrm{kg}$ a-asarone (Table 2). Therefore, these results indicate that a single acute administration of a-asarone had only a moderate effect on MES-induced seizures and death. Single acute administration of a-asarone had weak anticonvulsant activity on MES-induced seizures. No significant difference was observed among these treatments.

Effects of Chronic Treatment with a-Asarone on PTZInduced Seizures All animals $(n=20)$ treated with $85 \mathrm{mg} / \mathrm{kg}$ PTZ (treated with vehicle) had generalized seizures (Fig. 2A). The onset time of seizure was $237 \pm 44 \mathrm{~s}$ (Fig. 2C). Chronic

Table 1. Effect of Chronic Treatment with a-Asarone on MES-Induced Convulsions in Mice

\begin{tabular}{ccccc}
\hline \hline Treatment & Dose & $n$ & Seizure incidence (\%) & Mortality (\%) \\
\hline a-Asarone & $50 \mathrm{mg} / \mathrm{kg}$ & 20 & $40^{*}$ & $30^{*}$ \\
a-Asarone & $100 \mathrm{mg} / \mathrm{kg}$ & 20 & $20^{* *}$ & $10^{* *}$ \\
a-Asarone & $200 \mathrm{mg} / \mathrm{kg}$ & 20 & $0^{* *}$ & $0 * *$ \\
VPA & $200 \mathrm{mg} / \mathrm{kg}$ & 20 & $0^{* *}$ & $0 *$ \\
Vehicle & $10 \mathrm{~mL} / \mathrm{kg}$ & 20 & 100 & 40 \\
\hline
\end{tabular}

Data are presented as percentage of tonic seizures. Chronic a-asarone was applied for $28 \mathrm{~d}$; the last time was 60 min before electroconvulsions. ${ }^{*} p<0.05, * * p<0.01 v s$. vehicle-treated group. 
treatment with a-asarone at doses of 50,100 , and $200 \mathrm{mg} / \mathrm{kg}$ significantly decreased the incidence of seizure induced by PTZ in 50\% (10/20), 30\% (6/20), and 15\% (3/20) of tested animals, respectively, compared to $100 \%(20 / 20)$ in the vehicle treated group and $10 \%(2 / 20)$ in the CNP treated group $(p<0.01$, Fig. 2A). a-Asarone at doses of 50, 100, and $200 \mathrm{mg} /$ $\mathrm{kg}$ also significantly decreased the mortality induced by PTZ in $40 \%(8 / 20), 25 \%(5 / 20)$, and $10 \%(2 / 20)$ of tested animals, respectively, compared to $90 \%(20 / 20)$ in the vehicle treated group ( $p<0.01$, Fig. $2 \mathrm{~B}$ ). Chronic treatment with a-asarone at doses of 50,100 , and $200 \mathrm{mg} / \mathrm{kg}$ significantly increased the latency of seizure $(348 \pm 57 \mathrm{~s}$ for $50 \mathrm{mg} / \mathrm{kg}, 402 \pm 69 \mathrm{~s}$ for $100 \mathrm{mg} /$ $\mathrm{kg}$, and $422 \pm 78 \mathrm{~s}$ for $200 \mathrm{mg} / \mathrm{kg}$ ) compared to the vehicle treated group (237 $\pm 44 \mathrm{~s}$ ) (Fig. 2C). An ANOVA performed on the seizure latency revealed a significant group difference $[F=4.952, p<0.01]$. Chronic treatment with a-asarone significantly decreased the duration of seizures in a dose-dependent manner, and this effect was observed at all doses tested $(31.8 \pm 2.2 \mathrm{~s}$ for $50 \mathrm{mg} / \mathrm{kg}, 23.5 \pm 1.9 \mathrm{~s}$ for $100 \mathrm{mg} / \mathrm{kg}, 11.4 \pm$ $1.6 \mathrm{~s}$ for $200 \mathrm{mg} / \mathrm{kg}$ ) as compared to the vehicle treated group (42.4 $\pm 2.3 \mathrm{~s})$ (Fig. 2D). An ANOVA performed on seizure duration revealed a significant group difference $[F=5.562$, $p<0.01]$. CNP $(2 \mathrm{mg} / \mathrm{kg})$, the positive control, significantly increased the seizure latency, decreased the seizure duration, and prevented the death of animals when compared to vehicletreated animals $(p<0.01)$.

Effects of Acute Treatment with a-Asarone on PTZInduced Seizures In the control mice, subcutaneous PTZ $(85 \mathrm{mg} / \mathrm{kg})$ consistently induced clonic seizures followed by tonic seizures and death (Table 3). Oral administration of aasarone to mice at $50 \mathrm{mg} / \mathrm{kg}$ to $200 \mathrm{mg} / \mathrm{kg}$ did not prevent the occurrence of clonic seizures, tonic seizures, or death (Table 3). There was no difference between the number of animals that convulsed with any of the acute doses of a-asarone: $100 \%$ (20/20) for vehicle, $100 \%(20 / 20)$ for $50 \mathrm{mg} / \mathrm{kg}, 95 \%(19 / 20)$ for $100 \mathrm{mg} / \mathrm{kg}$, and $90 \%(18 / 20)$ for $200 \mathrm{mg} / \mathrm{kg}$, although the onsets of tonic seizures and death were significantly prolonged as compared with the control (Table 3). These results indicated that a single acute administration of a-asarone had only a moderate effect on PTZ-induced seizures and death. Single acute administration of a-asarone had weak anticonvulsant activity on PTZ-induced seizures.
Effects of Acute Treatment with a-Asarone on LI-PILO Induced SE Administration of $40 \mathrm{mg} / \mathrm{kg}$ pilocarpine $(n=20)$ at $18-24 \mathrm{~h}$ after injection of $125 \mathrm{mg} / \mathrm{kg}$ lithium chloride caused SE in $95 \%(19 / 20)$ of the animals in the saline group (treated with vehicle) (Fig. 3A). Pretreatment with a-asarone orally at a dose of 50,100 , or $200 \mathrm{mg} / \mathrm{kg}$ significantly decreased the occurrence of pilocarpine-induced SE in $80 \%(16 / 20), 60 \%$ $(12 / 20)$, and $50 \%(10 / 20)$ of tested animals, respectively, compared to $95 \%(19 / 20)$ in the vehicle treated group and $40 \%$ $(8 / 20)$ in the CBZ treated group (Fig. 3A). In the remaining rats in which continuous seizures were not suppressed, a-asarone pretreatment significantly delayed the onset of SE; this effect was observed at all doses tested $(25.37 \pm 4.60 \mathrm{~min}$ for $50 \mathrm{mg} / \mathrm{kg}, 31.20 \pm 5.42 \mathrm{~min}$ for $100 \mathrm{mg} / \mathrm{kg}, 36.42 \pm 8.14 \mathrm{~min}$ for $200 \mathrm{mg} / \mathrm{kg})$ as compared to the vehicle treated group $(21.50 \pm$ $6.69 \mathrm{~min}$ ) and CBZ treated group (41.18 $\pm 7.65 \mathrm{~min})$ (Fig. 3C). An ANOVA performed on the onset of SE revealed a significant group difference $[F=10.457, p<0.01]$. Pretreatment with a-asarone orally at a dose of 50,100 , and $200 \mathrm{mg} / \mathrm{kg}$ significantly decreased the mortality induced by pilocarpine in $30 \%(6 / 20), 20 \%(4 / 20)$, and $10 \%(2 / 20)$ of tested animals, respectively, compared to $50 \%(10 / 20)$ in the vehicle treated group and 5\% (1/20) in the CBZ treated group (Fig. 3B). Pretreatment with a-asarone also significantly reduced the severity of pilocarpine-induced seizures; this effect was observed at all doses tested, $50 \mathrm{mg} / \mathrm{kg}(3.38 \pm 1.63), 100 \mathrm{mg} / \mathrm{kg}(2.44 \pm 1.16)$, and $200 \mathrm{mg} / \mathrm{kg}(2.16 \pm 0.74)$ compared to the vehicle treated group (4.12 \pm 0.35$)$ and CBZ treated group (2.04 \pm 0.66$)$ (Fig. $3 \mathrm{D})$. An ANOVA performed on the seizure severity revealed a significant group difference $[F=11.396, p<0.01]$. The SE incidence, SE latency, and seizure severity as well as mortality were significantly reduced after treatment with a-asarone at different doses.

Effects of Chronic Treatment with a-Asarone on LIPILO Induced SRSs All the rats (100\%) studied until the chronic phase developed SRS with a latency of $15.8 \pm 2.5 \mathrm{~d}$ in the vehicle treated group (Fig. 4B). The incidence of SRSs during treatment was $75-95 \%$ in the $50-200 \mathrm{mg} / \mathrm{kg}$ a-asarone treated group and $70 \%$ in the CBZ-treated group (Fig. 4A). The latency for the first spontaneous seizure was $(16.5 \pm 2.7 \mathrm{~d}$, $17.4 \pm 2.8 \mathrm{~d}, 19.7 \pm 3.4 \mathrm{~d}$, and $20.6 \pm 3.2 \mathrm{~d}$ for the $50-200 \mathrm{mg} / \mathrm{kg}$ a-asarone groups and CBZ groups, respectively (Fig. 4B). An

Table 2. Acute Effects of a-Asarone on MES-Induced Convulsions in Mice

\begin{tabular}{crrr}
\hline \hline Treatment & \multicolumn{1}{c}{ Dose } & $n$ & Seizure incidence (\%) \\
\hline a-Asarone & $50 \mathrm{mg} / \mathrm{kg}$ & 20 & 100 \\
a-Asarone & $100 \mathrm{mg} / \mathrm{kg}$ & 20 & 95 \\
a-Asarone & $200 \mathrm{mg} / \mathrm{kg}$ & 20 & 90 \\
Vehicle & $10 \mathrm{~mL} / \mathrm{kg}$ & 20 & 40 \\
\hline
\end{tabular}

Data are presented as percentage of tonic seizures. Acute a-asarone was applied $60 \mathrm{~min}$ before electroconvulsions.

Table 3. Acute Effects of a-Asarone on PTZ-Induced Convulsions in Mice

\begin{tabular}{|c|c|c|c|c|c|c|}
\hline Treatment & Dose & $n$ & Seizure incidence $(\%)$ & Seizure latency (s) & Onset of death (s) & Mortality (\%) \\
\hline a-Asarone & $50 \mathrm{mg} / \mathrm{kg}$ & 20 & 100 & $260 \pm 31 *$ & $453 \pm 45^{*}$ & 85 \\
\hline a-Asarone & $100 \mathrm{mg} / \mathrm{kg}$ & 20 & 95 & $303 \pm 45 * *$ & $526 \pm 62 * *$ & 80 \\
\hline a-Asarone & $200 \mathrm{mg} / \mathrm{kg}$ & 20 & 90 & $325 \pm 51 * *$ & $542 \pm 78 * *$ & 80 \\
\hline Vehicle & $10 \mathrm{~mL} / \mathrm{kg}$ & 20 & 100 & $215 \pm 23$ & $375 \pm 40$ & 90 \\
\hline
\end{tabular}

Data are presented as percentage of clonic seizures or mean \pm S.E.M. Acute a-asarone was applied $60 \mathrm{~min}$ before the injection of PTZ $(85 \mathrm{mg} / \mathrm{kg}) . * p<0.05, * * p<0.01 v s$. vehicle-treated group. 

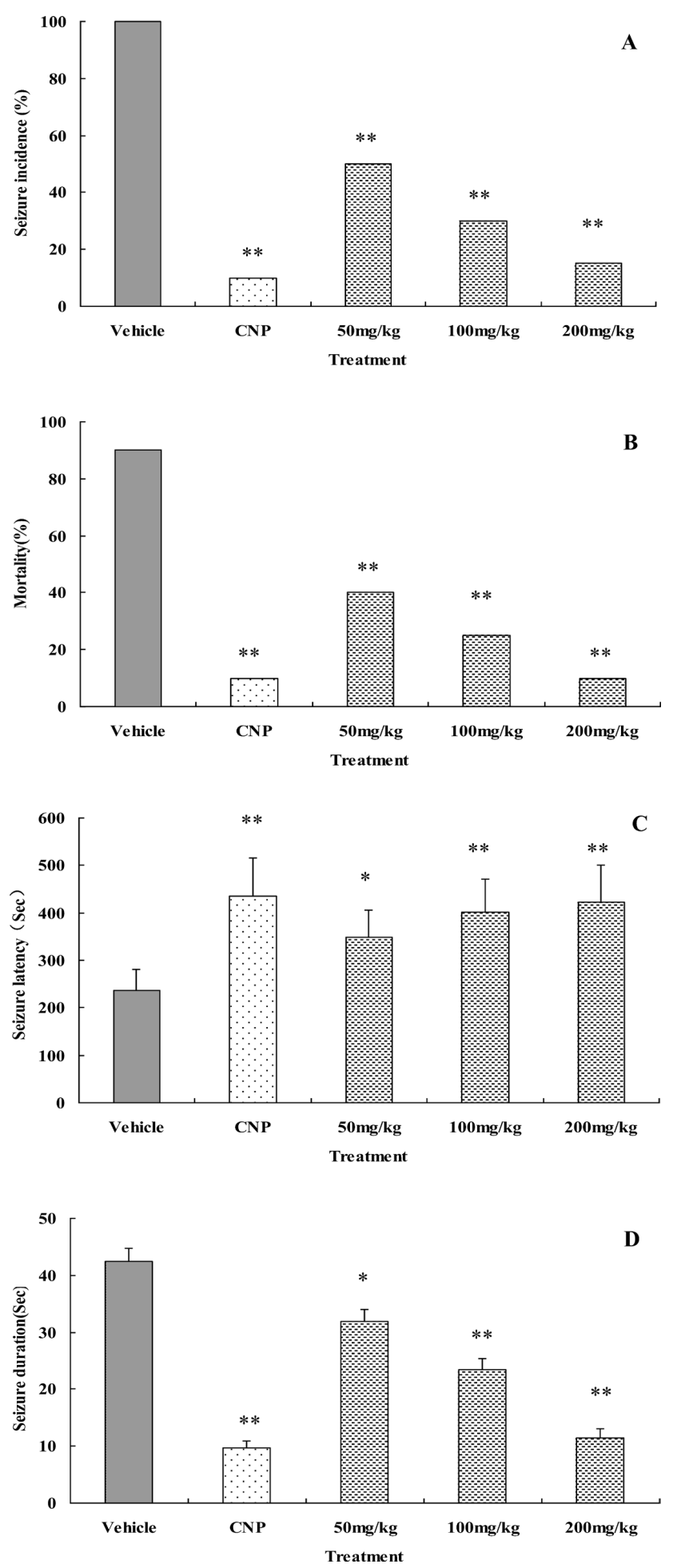

Fig. 2. Chronic Treatment with a-Asarone Alters the Expression of PTZ-Induced Clonic Seizures

(A) Chronic treatment with a-asarone $(50,100$, or $200 \mathrm{mg} / \mathrm{kg} / \mathrm{d}$, p.o. $)$ reduced the incidence of PTZ-induced clonic seizures. (B) Chronic treatment with a-asarone $(50,100$, or $200 \mathrm{mg} / \mathrm{kg} / \mathrm{d}$, p.o. $)$ reduced the mortality. (C) Chronic treatment with a-asarone $(50,100$, or $200 \mathrm{mg} / \mathrm{kg} / \mathrm{d}$, p.o. $)$ delayed the onset of PTZ-induced clonic seizures. (D) Chronic treatment with a-asarone (50, 100, or $200 \mathrm{mg} / \mathrm{kg} / \mathrm{d}$, p.o.) reduced the duration of PTZ-induced clonic seizures. Chronic a-asarone was applied for $28 \mathrm{~d}$; the last time was $60 \mathrm{~min}$ before the injection of PTZ $(85 \mathrm{mg} / \mathrm{kg}) *{ }^{*} p<0.05$, $* * p<0.01 v s$. vehicle-treated group.
ANOVA performed on the SRS latency revealed a significant group difference $[F=8.532, p<0.05]$. Seizure severity was $4.35 \pm 0.41$ during saline treatment, $4.02 \pm 0.39,3.37 \pm 0.34$, and $2.98 \pm 0.31$ during $50-200 \mathrm{mg} / \mathrm{kg}$ a-asarone treatment, and 2.66 \pm 0.32 during CBZ treatment, respectively (Fig. 4C). An ANOVA performed on the seizure severity revealed a significant group difference $[F=9.286, p<0.05]$. Seizure frequency was $14.26 \pm 5.16$ during saline treatment, $14.12 \pm 4.98,13.24 \pm$ 4.56 , and $10.28 \pm 4.36$ during $50-100 \mathrm{mg} / \mathrm{kg}$ a-asarone treatment, and 9.45 \pm 3.22 during CBZ treatment (Fig. 4D). An ANOVA performed on the seizure frequency revealed a significant group difference $[F=6.615, p<0.05]$. Higher doses of a-asarone $(100-200 \mathrm{mg} / \mathrm{kg})$ reduced the incidence of spontaneous recurrent seizures, seizure severity, and seizure frequency during treatment.

\section{DISCUSSION}

a-Asarone is a major essential oil component in rhizomes of Acorus gramineus, a traditional medicinal plant utilized in China to treat psychiatric disorders, narcosis and chronic tracheitis. a-Asarone has attracted widespread interest recently for its use as a potential antithrombotic, antimicrobial, insecticidal, nematicidal and antifeedant agent. ${ }^{27)}$ In addition, animal studies have demonstrated various pharmacological characteristics of a-asarone in the nervous system, such as an anticonvulsive action $^{28)}$ or sedative and hypothermic effects. ${ }^{29,30)}$ Moreover, Hu et al. found that a-asarone at a dose of $48 \mathrm{mg} / \mathrm{kg}$ intraperitoneally (i.p.) produced a significant decrease in locomotor activity and prolonged the pentobarbital sleeping time significantly in mice. ${ }^{31)}$ Taking into consideration the pharmacological profile of a-asarone, the present study focused on the anticonvulsant effects of chronic treatment with a-asarone.

The data obtained in the present study demonstrate that chronic treatment with a-asarone has a significant anticonvulsant effect on MES and PTZ and lithium-pilocarpine induced seizures. The present results suggest that the anticonvulsant effects of a-asarone are only observed after continuous daily oral administration of a-asarone (for at least 4 weeks). This confirms the previous findings that chronic preinhalation of an essential oil can reduce seizure severity in the PTZ mouse model. ${ }^{16)}$ The lack of an effect of acutely administered aasarone on seizure activity also demonstrates that a-asarone may not directly modify seizure activity. It appears that aasarone would not be a priori able to control acute seizures in epilepsy.

The MES is a model for generalized tonic-clonic seizures. It is highly reproducible with consistent endpoints. The behavioral and electrographic seizures generated in this model are consistent with the human disorder. ${ }^{32)}$ This model identifies those compounds which prevent seizure spread. Abolition of the hind limb tonic extensor component indicates inhibit MES-induced seizure spread. ${ }^{33)}$ The MES is a standard procedure that evaluates the ability of testing materials to protect against hind limb extension in MES. Toman and Swinyard reported that the seizure patterns in MES for all laboratory animals and humans are similar except for the time scale. ${ }^{34)}$ Protection against hind limb extension in the MES predicts the anticonvulsant activity of antiepileptic drugs that prevent the spread of an epileptic seizure from an epileptic focus during seizure activity. Protection against hind limb extension 

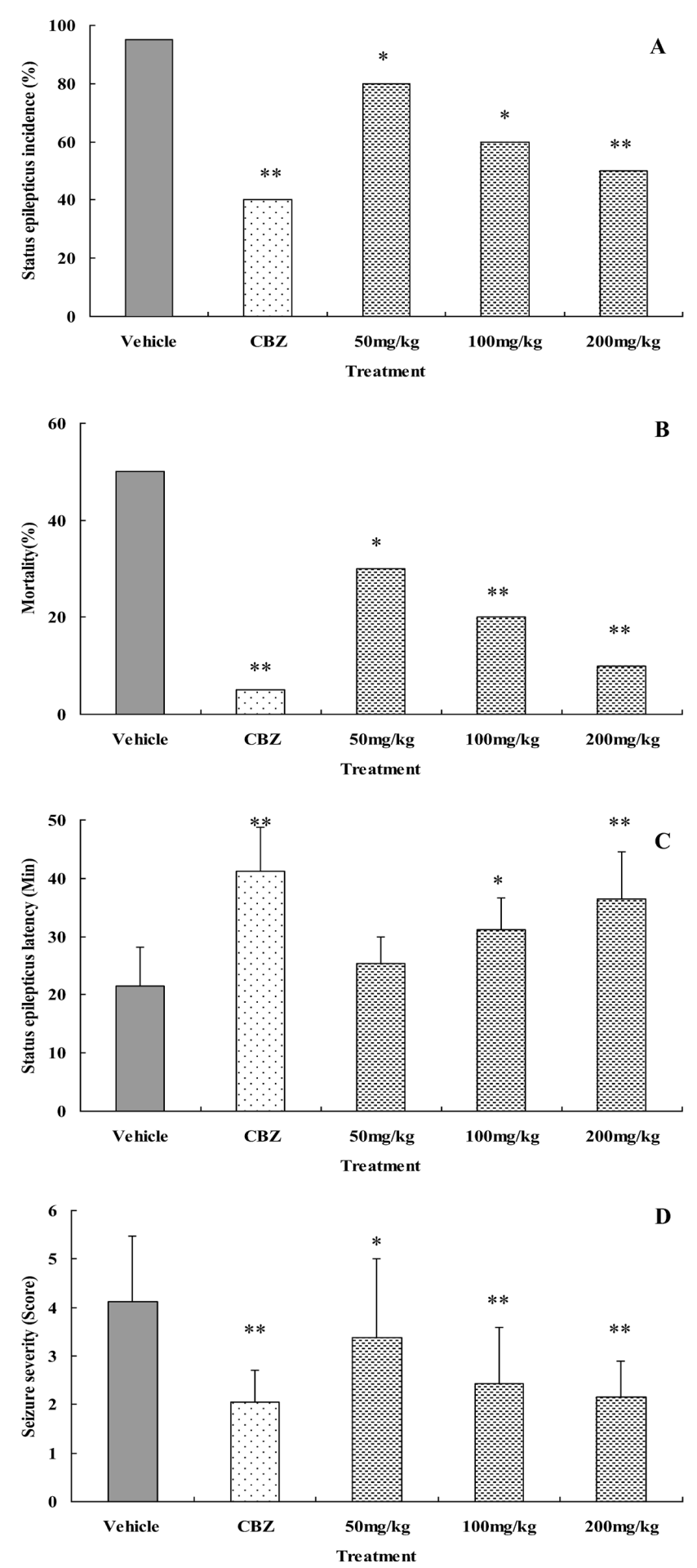

Fig. 3. a-Asarone Pretreatment Altered the Expression of PilocarpineInduced SE

(A) a-Asarone pretreatment $(50,100$, or $200 \mathrm{mg} / \mathrm{kg} / \mathrm{d}$, p.o. $)$ reduced the incidence of SE. (B) a-Asarone pretreatment $(50,100$, or $200 \mathrm{mg} / \mathrm{kg} / \mathrm{d}$, p.o. $)$ reduced the mortality. (C) a-Asarone pretreatment $(50,100$, or $200 \mathrm{mg} / \mathrm{kg} / \mathrm{d}$, p.o.) delayed the onset of pilocarpine-induced SE. (D) a-Asarone pretreatment $(50,100$, or $200 \mathrm{mg}$ / $\mathrm{kg} / \mathrm{d}$, p.o.) reduced the severity of pilocarpine-induced seizures. a-Asarone was orally administered $60 \mathrm{~min}$ before the injection of pilocarpine $(40 \mathrm{mg} / \mathrm{kg}) .{ }^{*} p<0.05$, $* * p<0.01 v s$. vehicle-treated group.
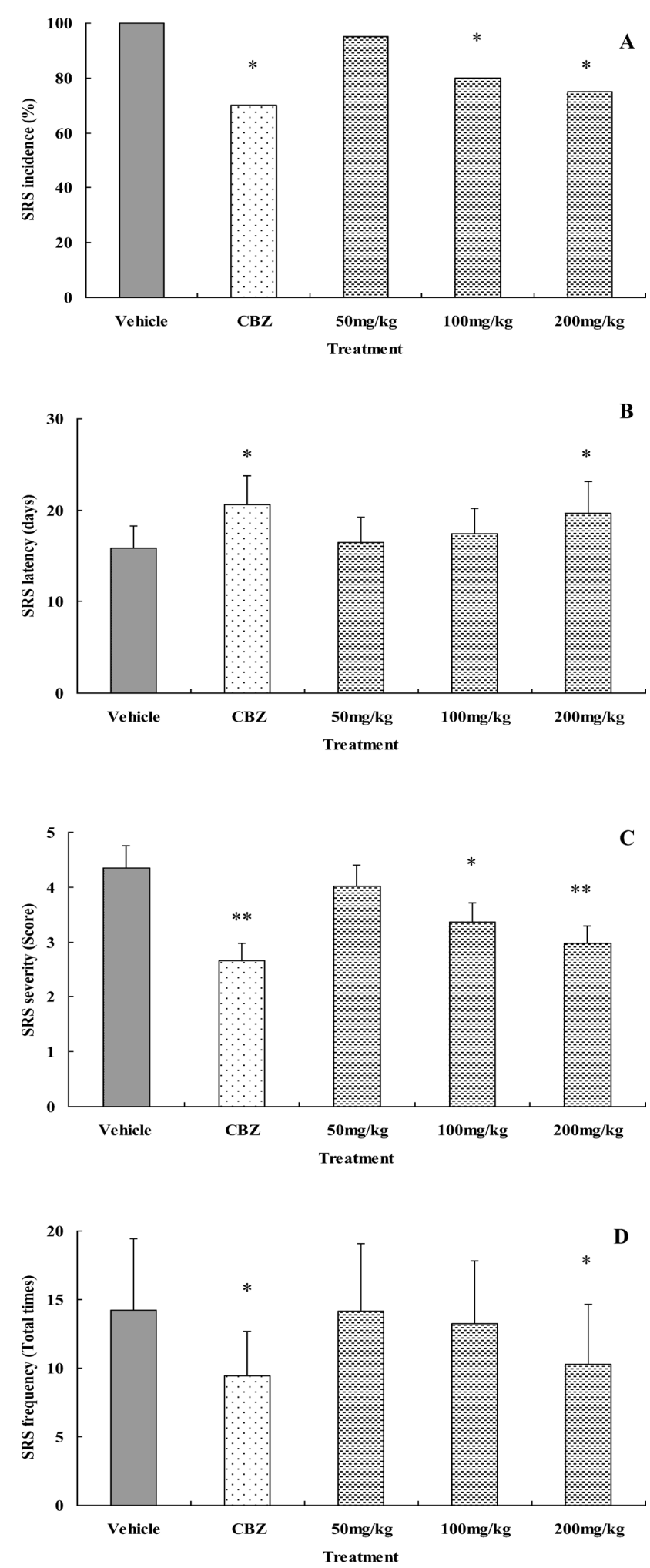

Fig. 4. Chronic Treatment with a-Asarone Altered the Expression of Pilocarpine-Induced SRSs

(A) Chronic treatment with a-asarone $(50,100$, or $200 \mathrm{mg} / \mathrm{kg} / \mathrm{d}$, p.o.) reduced the incidence of SRSs. (B) Chronic treatment with a-asarone $(50,100$, or $200 \mathrm{mg} / \mathrm{kg} / \mathrm{d}$, p.o.) delayed the onset of pilocarpine-induced SRSs. (C) Chronic treatment with aasarone $(50,100$, or $200 \mathrm{mg} / \mathrm{kg} / \mathrm{d}$, p.o. $)$ reduced the severity of pilocarpine-induced SRSs. (D) Chronic treatment with a-asarone $(50,100$, or $200 \mathrm{mg} / \mathrm{kg} / \mathrm{d}$, p.o.) reduced the frequency of pilocarpine-induced SRSs. Chronic a-asarone was applied for $28 \mathrm{~d}$. $* p<0.05, * * p<0.01 v s$. vehicle-treated group. 
also indicates the ability of the testing material to inhibit or prevent seizure discharge within the brain stem substrate. ${ }^{35)}$ In the MES test, a-asarone $(200 \mathrm{mg} / \mathrm{kg})$ completely abolished the hind limb tonic extension thereby providing 100\% seizure protection in mice chronically treated with this fraction. The effect of a-asarone on MES-induced seizures indicates that it may be effective in treating generalized tonic-clonic seizures.

The scPTZ test is a model that primarily identifies compounds that raise seizure threshold. The scPTZ test utilizes a dose of pentylenetetrazol $(85 \mathrm{mg} / \mathrm{kg}$ in mice). This produces clonic seizures lasting for a period of at least five seconds in 97 percent (CD97) of animals tested. With some minor exceptions, the pharmacological profile of the scPTZ seizure model is consistent with human condition. ${ }^{36)}$ The absence of clonic spasms in the observed time period indicates a compound's ability to abolish the effect of pentylenetetrazol on seizure threshold. ${ }^{37)}$ a-Asarone also exhibited a significant delayed onset of clonic-tonic actions and protection from PTZ-induced mortality. This finding for a-asarone may indicate its efficacy for myoclonic seizures.

The LI-PILO models have been rigorously explored as animal models of temporal lobe epilepsy. ${ }^{38)}$ LI-PILO models qualitatively reproduce in rodents many of the sequelae noted in human populations of temporal lobe epilepsy, including behavioral features [cognitive deficits, SRSs], electroencephalographic (EEG) characteristics, and the pattern of neuroanatomical damage. ${ }^{39)}$ In the rat, SE can be experimentally evoked by a single systemic injection of lithium chloride followed 18 or $24 \mathrm{~h}$ later by a muscarinic agonist. During the days following the induction of SE, a silent period begins and is characterized by normal EEG activity and behavior of the rodent; over the next several weeks, the rodent enters a chronic period characterized both behaviorally and electroencephalographically by the appearance of SRSs. ${ }^{40)}$ In this study, chronic treatment with a-asarone significantly reduced the incidence of spontaneous recurrent seizures, seizure severity, and seizure frequency. This finding for a-asarone indicates that a-asarone treatment during the silent period did not abort the epileptogenesis but minimized the expression of seizures.

In the present study, chronic administration of a-asarone to mice and rats at doses up to $100 \mathrm{mg} / \mathrm{kg} / \mathrm{d}$ also did not result in any toxic symptoms. Opposing results were obtained in previous studies where a-asarone was given i.p. to mice or rats in an acute test, and have confirmed the effects of this drug on general behavior such as decreased locomotor activity and potentiation of the pentobarbital-induced sleep time..$^{17,30,31)}$ Therefore, it was confirmed that variations in the route and period of administration are factors that determine the adverse effects of a-asarone. Of course, in view of species and administration schedule differences, further studies are necessary to investigate different aspects of the toxic and nontoxic effects of a-asarone. Whether the anticonvulsant effects of a-asarone are mediated by its sedative effects requires further investigation, although close examination of the behavioral records indicate no major behavioral differences between a-asarone treated and control animals.

In conclusion, a single acute administration of a-asarone produced weak anticonvulsant activity, but daily administration of a-asarone for 4 weeks diminished the convulsion and reduced the number of animals that died as a result of seizure induction. The results of this laboratory animal study indicate that a-asarone possesses multieffective potency and causes changes in brain function after chronic treatment. However, the mechanism by which a-asarone produces these effects is presently unknown and further studies are required to clarify the exact mechanism related to the therapeutic efficacy of a-asarone. The data obtained from our study lend pharmacological credence to the suggested ethnomedical uses of Acorus gramineus in the management of convulsions and epilepsy in China.

Acknowledgements This study was in part supported by a Chongqing Medical University Doctoral Scholarship. We would like to express our sincere thanks to Professor Zhang Mao for her skillful technical advice and support. The authors also wish to thank Dr. Duan QL of Shanghai Tongji University for his help with editing the manuscript.

\section{REFERENCES}

1) Remy S, Beck H. Molecular and cellular mechanisms of pharmacoresistance in epilepsy. Brain, 129, 18-35 (2006).

2) Granata $T$, Marchi N, Carlton E, Ghosh C, Gonzalez-Martinez $\mathrm{J}$, Alexopoulos AV, Janigro D. Management of the patient with medically refractory epilepsy. Expert Rev. Neurother., 9, 1791-1802 (2009).

3) Dalkara S, Karakurt A. Recent progress in anticonvulsant drug research: strategies for anticonvulsant drug development and applications of antiepileptic drugs for non-epileptic central nervous system disorders. Curr. Top. Med. Chem., 12, 1033-1071 (2012).

4) Spinella M. Herbal medicines and epilepsy: the potential for benefit and adverse effects. Epilepsy Behav., 2, 524-532 (2001).

5) Liu GQ, Sun JN, He ZZ, Jiang Y. Spasmolytic actions of active principles of volatile of Acorus gramineus (Chinese). Acta Pharmacol. Sin., 4, 95-97 (1983).

6) Vohora SB, Shah SA, Dandiya PC. Central nervous system studies on an ethanol extract of Acorus calamus rhizomes. J. Ethnopharmacol., 28, 53-62 (1990).

7) Liao WP, Chen L, Yi YH, Sun WW, Gao MM, Su T, Yang SQ. Study of antiepileptic effect of extract from acorus tatarinowii schott. Epilepsia, Suppl. 1, S21-S24 (2005).

8) Liao JF, Huang SY, Jan YM, Yu LL, Chen CF. Central inhibitory effects of water extract of Acori graminei rhizoma in mice. J. Ethnopharmacol., 61, 185-193 (1998).

9) Nishiyama N, Zhou Y, Takashina K, Saito H. Effects of DX-9386, a traditional Chinese preparation, on passive and active avoidance performances in mice. Biol. Pharm. Bull., 17, 1472-1476 (1994).

10) Lee B, Choi Y, Kim H, Kim SY, Hahm DH, Lee HJ, Shim I. Protective effects of methanol extract of Acori graminei rhizoma and Uncariae RAmulus et Uncus on ischemia-induced neuronal death and cognitive impairments in the rat. Life Sci., 74, 435-450 (2003).

11) Du Z, Clery RA, Hammond CJ. Volatiles from leaves and rhizomes of fragrant Acorus spp. (Acoraceae). Chem. Biodivers., 5, 887-895 (2008).

12) Dai J, Ha C, Shen M. Systematic study of $\beta$-asarone-rich volatile oil from Acori graminei rhizoma by off-line supercritical $\mathrm{CO}_{2}$ extraction-gas chromatography-mass spectrometry. J. Sep. Sci., 31, 714-720 (2008)

13) Limón ID, Mendieta L, Díaz A, Chamorro G, Espinosa B, Zenteno E, Guevara J. Neuroprotective effect of alpha-asarone on spatial memory and nitric oxide levels in rats injected with amyloid- $\beta$ (25-35). Neurosci. Lett., 453, 98-103 (2009).

14) Zhang H, Han T, Yu CH, Rahman K, Qin LP, Peng C. Ameliorating effects of essential oil from Acori graminei rhizoma on learning and memory in aged rats and mice. J. Pharm. Pharmacol., 59, 301-309 (2007). 
15) Cho J, Kim YH, Kong JY, Yang CH, Park CG. Protection of cultured rat cortical neurons from excitotoxicity by asarone, a major essential oil component in the rhizomes of Acorus gramineus. Life Sci., 71, 591-599 (2002).

16) Koo BS, Park KS, Ha JH, Park JH, Lim JC, Lee DU. Inhibitory effects of the fragrance inhalation of essential oil from Acorus gramineus on central nervous system. Biol. Pharm. Bull., 26, 978-982 (2003).

17) Pages N, Maurois P, Delplanque B, Bac P, Stables JP, Tamariz J, Chamorro G, Vamecq J. Activities of $\alpha$-asarone in various animal seizure models and in biochemical assays might be essentially accounted for by antioxidant properties. Neurosci. Res., 68, 337-344 (2010).

18) Lorke D. A new approach to practical acute toxicity testing. Arch. Toxicol., 54, 275-287 (1983).

19) Tedeschi DH, Swinyard EA, Goodman LS. Effects of variations in stimulus intensity on maximal electroshock seizure pattern, recovery time, and anticonvulsant potency of phenobarbital in mice. $J$. Pharmacol. Exp. Ther., 116, 107-113 (1956).

20) White HS, Johnson M, Wolf HH, Kupferberg HJ. The early identification of anticonvulsant activity: role of the maximal electroshock and subcutaneous pentylenetetrazol seizure models. Ital. J. Neurol. Sci., 16, 73-77 (1995).

21) Eells JB, Clough RW, Browning RA, Jobe PC. Fos in locus coeruleus neurons following audiogenic seizure in the genetically epilepsyprone rat: comparison to electroshock and pentylenetetrazol seizure models. Neurosci. Lett., 233, 21-24 (1997).

22) Turski WA, Cavalheiro EA, Schwarz M, Czuczwar SJ, Kleinrok Z, Turski L. Limbic seizures produced by pilocarpine in rats: behavioural, electroencephalographic and neuropathological study. Behav. Brain Res., 9, 315-335 (1983).

23) Honchar MP, Olney JW, Sherman WR. Systemic cholinergic agents induce seizures and brain damage in lithium-treated rats. Science, 220, 323-325 (1983).

24) Müller CJ, Bankstahl M, Gröticke I, Löscher W. Pilocarpine vs. lithium-pilocarpine for induction of status epilepticus in mice: development of spontaneous seizures, behavioral alterations and neuronal damage. Eur. J. Pharmacol., 619, 15-24 (2009).

25) Racine RJ. Modification of seizure activity by electrical stimulation. II. Motor seizure. Electroencephalogr. Clin. Neurophysiol., 32, 281-294 (1972).

26) Leite JP, Cavalheiro EA. Effects of conventional antiepileptic drugs in a model of spontaneous recurrent seizures in rats. Epilepsy Res., 20, 93-104 (1995).

27) Medina-Franco JL, Lopez-vallejo FL, Rodriguez-Morales S,
Castillo R, Chamorro G, Tamariz J. Molecular docking of the highly hypolipidemic agent a-asarone with the catalytic portion of HMG-CoA reductase. Bioorg. Med. Chem. Lett., 4, 989-994 (2005).

28) Sharma JD, Dandiya PC, Baxter RM, Kandel SI. Pharmacodynamical effects of asarone and $\beta$-asarone. Nature, 192, 1299-1300 (1961).

29) Zanoli P, Avallone R, Baraldi M. Sedative and hypothermic effects induced by $\beta$-asarone, a main component of Acorus calamus. Phytother. Res., 12 (Suppl. 1), 114-116 (1998).

30) Dandiya PC, Menon MK. Effects of asarone and $\beta$-asarone on conditioned responses, fighting behaviour and convulsions. Br. Pharmacol. Chemother., 20, 436-442 (1963).

31) $\mathrm{Hu}$ JG, Gu J, Wang ZW. Central inhibitory effects of Acrous gramineus and its component in mice. Pharmacol. Clin. Chin. Mater. Med., 15, 19-21 (1999).

32) Löscher W, Fassbender CP, Nolting B. The role of technical, biological and pharmacological factors in the laboratory evaluation of anticonvulsant drugs. II. Maximal electroshock seizure models. Epilepsy Res., 8, 79-94 (1991).

33) White HS. Clinical significance of animal seizure models and mechanism of action studies of potential antiepileptic drugs. Epilepsia, Suppl. 1, S9-S17 (1997).

34) Toman JE, Swinyard EA. Some properties of experimental electroshock seizures. Proc. Am. Fed. Clin. Res., 2, 98-99 (1945).

35) Laffan RJ, Swinyard EA, Goodman LS. Stimulus intensity, maximal electroshock seizures, and potency of anticonvulsants in rats. Arch. Int. Pharmacodyn. Ther., 111, 60-69 (1957).

36) Löscher W, Hönack D, Fassbender CP, Nolting B. The role of technical, biological and pharmacological factors in the laboratory evaluation of anticonvulsant drugs. III. Pentylenetetrazole seizure models. Epilepsy Res., 8, 171-189 (1991).

37) Löscher W, Schmidt D. Which animal models should be used in the search for new antiepileptic drugs? A proposal based on experimental and clinical considerations. Epilepsy Res., 2, 145-181 (1988).

38) Curia G, Longo D, Biagini G, Jones RS, Avoli M. The pilocarpine model of temporal lobe epilepsy. J. Neurosci. Methods, 172 , 143-157 (2008).

39) André V, Dubé C, François J, Leroy C, Rigoulot MA, Roch C, Namer IJ, Nehlig A. Pathogenesis and pharmacology of epilepsy in the lithium-pilocarpine model. Epilepsia, Suppl. 5, S41-S47 (2007).

40) Furtado MA, Castro OW, Del Vecchio F, de Oliveira JA, GarciaCairasco N. Study of spontaneous recurrent seizures and morphological alterations after status epilepticus induced by intrahippocampal injection of pilocarpine. Epilepsy Behav., 20, 257-266 (2011). 